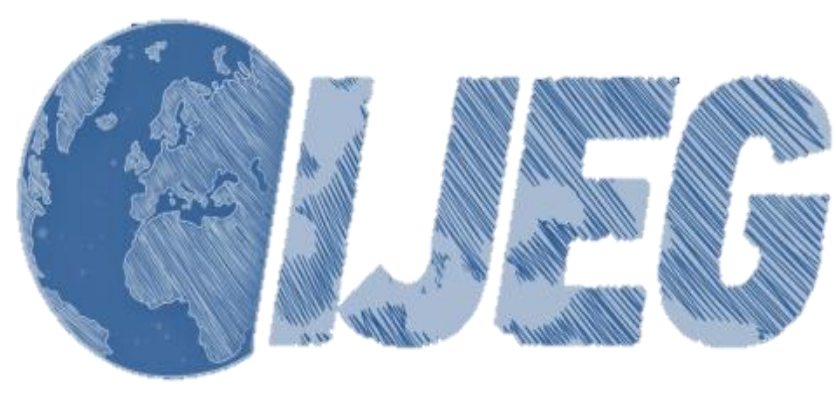

International Journal of Engineering and Geosciences (IJEG), Vol; 5, Issue; 3, pp. 150-159, October, 2020, ISSN 2548-0960, Turkey, DOI: 10.26833/ijeg.649961

\title{
ANECHOIC CHAMBER MEASUREMENTS FOR CIRCULAR ISAR IMAGING AT MERSIN UNIVERSITY'S MEATRC LAB
}

\author{
Sevket Demirci ${ }^{1 *}$, Caner Ozdemir ${ }^{1}$ \\ ${ }^{1}$ Mersin University, Engineering Faculty, Department of Electrical and Electronics Engineering, Mersin, Turkey \\ (sdemirci/cozdemir@mersin.edu.tr); ORCID 0000-0002-3020-7067, ORCID 0000-0003-2615-4203
}

*Corresponding Author, Received: 22/11/2019, Accepted: 29/01/2020

\begin{abstract}
Inverse synthetic aperture radar (ISAR) imaging is a reliable detection and classification technique for maneuvering targets at near and far-field ranges. In this study, we examine the near-field circular (turntable) ISAR imaging by conducting various real measurement experiments that were performed in the microwave anechoic chamber of the Mersin University's MEATRC laboratory. The backscattered data were collected via a vector network analyzer that works as a Stepped Frequency Continuous Wave (SFCW) radar and for a number of simple and complex metal objects. The collected raw data were calibrated by using the backscattering data of a canonical object and then focused by applying a near-field backprojection image reconstruction algorithm. The resultant circular ISAR images demonstrate successful and well localized detection of various types of targets even though they are camouflaged by clothing. The obtained results reveal the preliminary efficacy of $\mathrm{C}$ band ISAR imaging in concealed object detection problem encountered at security checkpoints such as airports.
\end{abstract}

Keywords: ISAR imaging, Near-field ISAR, Anechoic chamber, Concealed object detection 


\section{INTRODUCTION}

Inverse Synthetic Aperture Radar (ISAR) is a microwave remote sensing tool that uses target motion for the generation of an image of that target (Ozdemir, 2012). Aircrafts, helicopters, drones, ships and vehicles are amongst the common targets of ISAR imaging. In its basic mode, a two-dimensional (2D) ISAR image represents the estimate of the projection of the target's spatial reflectivity on a plane. Usually, this reflectivity map is largely dominated by contributions from some main components, termed as scattering centers. ISAR imagery has a prominent aspect in successfully exhibiting these hot points which have a critical role in target recognition (Ozdemir, 2012).

An important parameter in this context is the radar cross section (RCS) which determines the detectability metric of a target to a radar (Currie, 1989, Mensa, 1991 and Knott, 2006). A larger RCS indicates that the target can be more easily detected. Another important parameter is the resolution which defines the ability of radar to differentiate between the scattering centers. Range resolution is achieved by varying the frequency of the transmitted pulse, thus it depends on the frequency bandwidth. Cross-range resolution is obtained by varying the aspect of the target (i.e. changing the relative platform-target distance), therefore it depends on the angular data collection span. Obviously, wide angular spans improve cross-range resolution (Wehner, 1994).

Circular (or turntable) ISAR is a subcategory of ISAR which offers an effective way of utilizing the abovementioned parameters, i.e. RCS and resolution (Chen, et al 1980 and Kempf, et al 2007). To clarify, first note that circular ISAR incorporates the circular movement of a target, providing an observation coverage up to $360^{\circ}$. Hence, higher resolution imagery than that of narrow-angle ISAR is usually attained (Soumekh, 1999). Second, it can characterize anisotropic reflectivity mechanisms of the whole target, again due to its wideangle feature. Therefore, it is a well-suited research and validation tool for investigating targets' scattering behaviors.

Owing to these distinct features, circular ISAR has been widely deployed in numerous tasks. For instance, in RCS prediction and imaging of military targets, the measurements are often made in RCS chamber rooms or outdoor environment by placing targets on a rotating turntable. The obtained images are then examined for the purpose of lowering the overall RCS of targets via identifying and modifying the target structure to reduce the scattered energy in a given direction (Baird, et al 2005 , Kempf, et al 2007 and To, et al 2009). In nondestructive testing, like assessment of the deterioration of concrete structures, circular ISAR is mostly exploited to achieve high resolution maps of the material defects (Hong, et al 2000). In security applications such as imaging of concealed objects (Detlefsen, et al 2005, Bertl, et al 2007, Bertl, et al 2008, Bertl, et al 2010 and Sheen, et al 2010), a circular SAR or ISAR scanning is frequently used to obtain different views of the human body as well as in biomedical applications such as detection of tumors (Naseri, 2015, Avsar, et al 2016, Bicer, et al 2018 and Celik, et al 2019).

Besides its aforementioned advantages, circular ISAR has some challenges that need to be addressed for a successful application. For instance, the data collection geometry inherently presents a near-field inverse scattering problem which implies the requirement of a complex image reconstruction algorithm. Furthermore, the data collection and calibration processes should be accurately performed to benefit from circular ISAR's full potential as being very sensitive to errors.

In this paper, we aim to characterize various attributes of $2 \mathrm{D}$ circular ISAR imaging through microwave anechoic chamber data. Our focus is towards the $\mathrm{S}$ to $\mathrm{Ku}$ band imaging of various metal objects in order to provide a research resource for the concealed object detection problem. In our previous studies (Cetinkaya, et al 2011, Demirci, et al 2011, Demirci, et al 2012 and Demirci, et al 2013), this problem was approached by using millimeter wave band data. Herein, we evaluate the effectiveness of low frequency (up to $22 \mathrm{GHz}$ ) usage in penetrating through clothing camouflage. For this purpose, a series of experiments were conducted within the anechoic chamber room of the Advanced Technologies Research Center (MEATRC) laboratory at Mersin University. The calibrated images of the targets are reconstructed via near-field backprojection algorithm and analyzed in terms of resolution, far-field RCS, scattering features, frequency/angle sampling and effects of clothing obscure. The findings of these analyses and detailed discussions will be presented.

\section{THEORY FOR CIRCULAR ISAR IMAGING}

It is first worth noting that there are two key elements of a $2 \mathrm{D}$ radar reflection measurement geometry, i.e. range and cross-range. Range is the distance along the axis in the direction of the propagation of the beam whereas cross-range (or azimuth) is defined orthogonal to the range direction. As stated previously, frequency and angular diversities determine the resolutions in range and cross-range directions, respectively.

Circular ISAR takes into account the circular movement of either target or antenna to collect target's reflection information at different angular views. This can be achieved by moving the antenna on a target-centered circle or by rotating the target. Fig. 1 shows the 2D imaging geometry for the former case. The transceiver antenna transmits and receives radar waveforms, step by step, at the uniform samples of the azimuth angle positions $\emptyset$. As the look direction changes, the different geometrical information about the target can be acquired.

For such wide-angle data collections, plane-wave illumination of the whole target is usually not satisfied. The image reconstruction algorithms, therefore, should consider wavefront curvature effects to obtain focused imagery. One possible option could be the sub-aperture method which assumes planar wavefronts in smaller subapertures, thereby having a drawback of resolution degradation (Ozdemir, et al 2009). Another option could be the use of the backprojection algorithm (Munson, et al 1983 and Knaell et al 1995) which we have employed within this study. In the following, we briefly describe the formulation of this algorithm for a near-field data collection geometry.

With regard to the Fig. 1, let the target to be imaged is represented by a reflectivity function $g(x, y)$ and is assumed to be in the near-field of the antenna. The instantaneous look-angle $\varnothing$ is defined by the unit vector $\vec{u}$ which points from the origin towards the antenna. At each viewpoint, radar measures the range profile $d_{\emptyset}(r)$ 
given by

$d_{\emptyset}(r)=\int_{-\infty}^{\infty} \int_{-\infty}^{\infty} g(x, y) \delta\left(r_{u}-r\right) d x d y$

where $r_{u}$ stands for the near-field range from a viewpoint to any location $(x, y)$. Eqn. (1) is also known as Radon Transform of the scene which represents a projection of a three-dimensional (3D) target onto a one-dimensional (1D) function. ISAR systems generally acquire Fourier Transform (FT) data of this range profile which is expressed as

$D_{\emptyset}\left(k_{r}\right)=\int_{-\infty}^{\infty} d_{\emptyset}(r) \exp \left(-j k_{r} r\right) d r$

where $k_{r}$ is the wavenumber defined as $k_{r}=4 \pi f / c, f$ is the frequency and $c$ is the speed of light in vacuum. Stepped-frequency continuous wave (SFCW) radars measure the samples of this FT data at discrete frequency steps within a bandwidth $B$;

Backprojection algorithm benefits from the projection-slice theorem (Munson, et al 1983), whereby the measured $\boldsymbol{D}_{\varnothing}\left(\boldsymbol{k}_{\boldsymbol{r}}\right)$ can be linked to the target's FT $\boldsymbol{G}\left(\boldsymbol{k}_{x}, \boldsymbol{k}_{\boldsymbol{y}}\right)$ via $\boldsymbol{D}_{\emptyset}\left(\boldsymbol{k}_{\boldsymbol{r}}\right)=\boldsymbol{G}_{\emptyset}\left(\boldsymbol{k}_{\boldsymbol{r}}\right)$. Hence, a sampled representation of $\boldsymbol{G}\left(\boldsymbol{k}_{\boldsymbol{x}}, \boldsymbol{k}_{\boldsymbol{y}}\right)$ can be obtained from the projections $\boldsymbol{d}_{\emptyset}(\boldsymbol{r})$ measured at various observation angles. The algorithm uses this fact when utilizing the FT relationship between $\boldsymbol{g}(\boldsymbol{x}, \boldsymbol{y})$ and $\boldsymbol{G}\left(\boldsymbol{k}_{\boldsymbol{x}}, \boldsymbol{k}_{\boldsymbol{y}}\right)$. Then, the resulted equation for an estimate of $\boldsymbol{g}(\boldsymbol{x}, \boldsymbol{y})$ is found as

$g(x, y)=\int_{-\pi}^{\pi}\left[\int_{0}^{\infty} D_{\emptyset_{u}}\left(k_{r}\right) \exp \left(j k_{r} r_{u}\right) k_{r} d k_{r}\right] d \emptyset_{u}$

where the bracketed term in Eqn. (3) is the 1D filtered backprojection at the angle $\phi_{u}$. Denoting this by $q_{\emptyset_{u}}\left(r_{u}\right)$, it can be simply calculated by weighting the FT of the range profile $D_{\emptyset_{u}}\left(k_{r}\right)$ with a high-frequency filter $\left(k_{r}\right)$, and then by taking the 1D inverse Fourier transform (IFT). The consequential projections for different azimuth angles are then added to reconstruct an image of $g(x, y)$. In the implementation, a 1D interpolation procedure is required in image domain when the value of $q_{\emptyset_{u}}\left(r_{u}\right)$ is obtained for each pixel. At this backprojection step, if the true vector magnitudes of the nearfield ranges $r_{u}$ are taken, then the algorithm compensates the wavefront curvature effects. The reader is referred to (Demirci, et al, 2012) for a detailed formulation of this image reconstruction procedure.

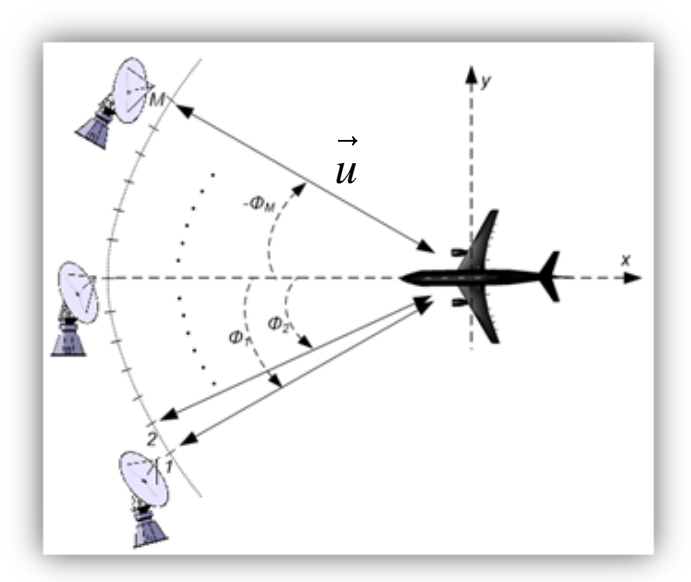

Figure.1 The geometry for 2D circular ISAR imaging (Ozdemir, 2012).

\subsection{Sampling Constraints}

Since the backscattering data from a SFCW radar are acquired through sampling of frequency $f$ and azimuth angle $\varnothing$, the characteristics of the resulting image are limited by these parameters. To avoid aliasing, the sampling intervals along each dimension should satisfy the Nyquist sampling criterion. These requirements for a circular ISAR scanning become as the followings:

$\Delta f \leq \frac{c}{2 R_{\max }}$

and

$\Delta \emptyset \leq \frac{\lambda_{\min }}{4 \rho_{\max }}$

where $\Delta \boldsymbol{f}$ is the frequency step, $\Delta \emptyset$ is the angular step, $\lambda_{\min }$ is the minimum transmitted wavelength, $\boldsymbol{R}_{\max }$ is the maximum unambigious range and $\boldsymbol{\rho}_{\max }$ is the target's maximum distance in radial direction.

\subsection{Resolutions}

Spatial resolutions in ISAR images depend on the frequency bandwidth, center frequency, extent of the angular view, as well as targets' scattering characteristics. In narrow-angle case, the range and cross-range resolutions are defined according to a separable $2 \mathrm{D}$ coordinate system. In wide-angle case, these resolutions, however, become no longer independent and the traditional formulas cannot be readily applied. Finding an analytical solution is also known to be a difficult task for this situation. Consequently, the resolutions of wideangle collections are usually computed via approximate methods. Herein, we adopted a method that is based on measurement of the 2D image area occupied the mainlobe return from a point scatterer. The method can be implemented with the following steps:

i. Obtain ISAR image of a point-like scatterer for a given $(\boldsymbol{f}, \emptyset)$ data set.

ii. Extract and display $\mathbf{- 4} \mathrm{dB}$ contour plot through the peak of target data. 
iii. Calculate $\mathbf{- 4} \mathrm{dB}$ resolutions in range and cross-range as the distances between the points where the peak value has fallen by $4 \mathrm{~dB}$.

The obtained values can then be used for evaluating the resolution performance of wide-angle ISAR data collection and image reconstruction procedure.

\section{OPERATION PRINCIPLES OF THE EXPERIMENTS}

\subsection{Experimental System}

Fig. 2 shows the schematic diagram of our experimental system used for reflection measurements within an anechoic chamber. It involves a vector network analyzer (VNA) with a SFCW operation, a transmitter (TX) and a receiver (RX) horn antenna in a quasimonostatic mode and a turntable. Quasi-monostatic means antennas are co-located with a slight separation between them. The target to be observed is placed on a Styrofoam support on the top of the turntable, at a range distance $R_{0}$ away from the midpoint of the antennas. The centers of antennas and the target are all located at the same height above the ground level with zero incidence angle. The turntable angle $\varnothing$ can be varied from $0^{\circ}$ to $360^{\circ}$ with a resolution of $\Delta \emptyset=0.1^{\circ}$. At each angle, the turntable is stopped and the scattering response at that angle is collected by VNA for a selected frequency bandwidth. The process is then repeated for different angles by rotating the turntable to the next angular position. A control computer with a customized MATLAB program communicates with VNA and position controller to control the rotation angle, measurement trigger and data logging. Thus, the automated measurements at multiple observation angles could be achieved at high sensitivity.

\subsection{Calibration Method}

Data calibration plays a crucial role in research and validation to minimize the undesired echoes. In this study, we have employed background subtraction procedure to calibrate the frequency domain backscatter data captured by a SFCW radar. In this procedure, two measurements are first performed with reference to the set-up in Fig. 2. One is for the actual target and the other one is for the canonical target that are both located at the center of the turntable with a normal incidence alignment. Later, a target-free measurement for the chamber room and support materials is performed. Using the theoretical backscattering data from a canonical target, the calibrated data can then be obtained as

$$
E_{S}(f)=\frac{E_{S}^{t a r}(f)-E_{S}^{b k g}(f)}{E_{S}^{c a l}(f)-E_{S}^{b k g}(f)} E_{S}^{c a l, \text { theo }}(f)
$$

where $E_{S}^{t a r}$ denotes the target's raw data to be calibrated, $E_{S}^{b k g}$ denotes the measured backscatter from chamber room without the target, $E_{S}^{c a l}$ and $E_{S}^{\text {cal,theo }}$ denote, respectively, the theoretical and measured backscatter from a canonical (i.e. reference) target and $E_{S}(f)$ denotes the calibrated data. It must be noted that, prior to the measurements, VNA is also internally calibrated at the ends of the cables by means of a 2-port response and isolation measurements.

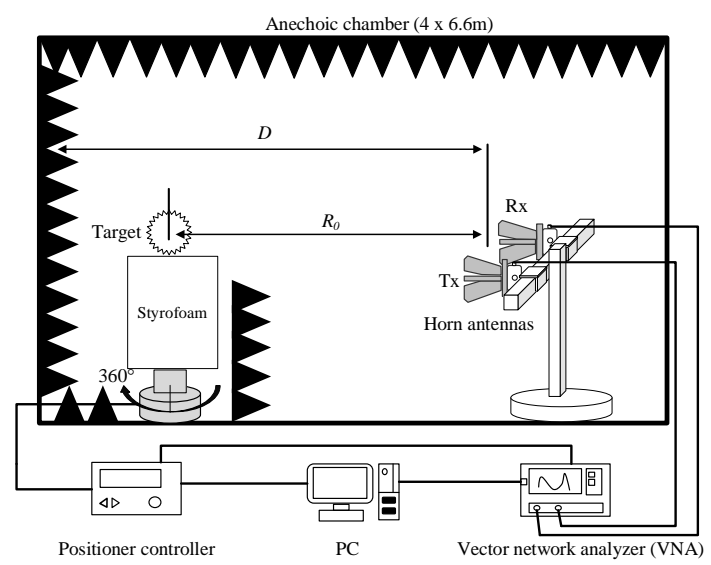

Figure.2 Illustration for the working principle of circular ISAR experiments.

\section{EXPERIMENTAL RESULTS}

\subsection{Reflectivity of chamber walls}

Before performing the imaging experiments, we first evaluated the performance of our anechoic chamber. A simple procedure explained in (Cottard, et al 2006) was utilized to obtain the reflectivity of the radar absorbing material that was used on the walls of the chamber. Using the geometry in Fig. 2 and locating a reference target, namely a metal plate with a size of $(a \times b)=(0.23 \mathrm{~m} \times$ $0.17 \mathrm{~m}$ ) on the Styrofoam box, backscattered data were collected for frequencies ranging from $4.5 \mathrm{GHz}$ to 8.5 GHz. The plate was placed perpendicular to the radar beam and precisely aligned pointing towards the midpoint of the line segment between the TX and RX antenna, laying at a distance of $R_{0}=2.64 \mathrm{~m}$ from this point. Afterwards, a similar measurement was carried out for the absorber at a stand-off distance of $D=2.2 \mathrm{~m}$. The measured reflectivity level was then computed via (Cottard, et al 2006).

$\sigma_{0}^{a b s o r b}=\left(\frac{1}{\pi R^{2}}\right) \sigma^{r e f}\left|\frac{E_{s}^{a b s o r b}}{E_{s}^{r e f}}\right|^{2}\left(\frac{D}{R}\right)^{4}$

where $\boldsymbol{\sigma}_{\mathbf{0}}^{\boldsymbol{a} \boldsymbol{b s o r b}}$ is the reflectivity (i.e., normalized RCS) of the absorber, $\sigma^{r e f}=\mathbf{4} \pi \boldsymbol{a}^{2} b^{2} / \lambda_{c}^{2}$ is the RCS of the metal plate with $\lambda_{\boldsymbol{c}}$ being the center wavelength and $\boldsymbol{E}_{\boldsymbol{s}}^{\boldsymbol{a} b s o r b}$ and $\boldsymbol{E}_{\boldsymbol{s}}^{\boldsymbol{r e f}}$ are the frequency domain data measured for the absorber and reference targets, respectively. Fig. 3(a) shows the measured range profiles obtained by taking 1D IFT of the frequency-domain data. As seen from the target's plot, the metal plate is detected at the correct range. Fig. 3(b) shows the measured reflectivity in $\mathrm{dB}$, plotted as a function of frequency. It is seen that the reflectivity level varies between $\mathbf{- 4 0} \mathrm{dB}$ and $\mathbf{- 7 0} \mathrm{dB}$ indicating an acceptable absorber characteristic. 


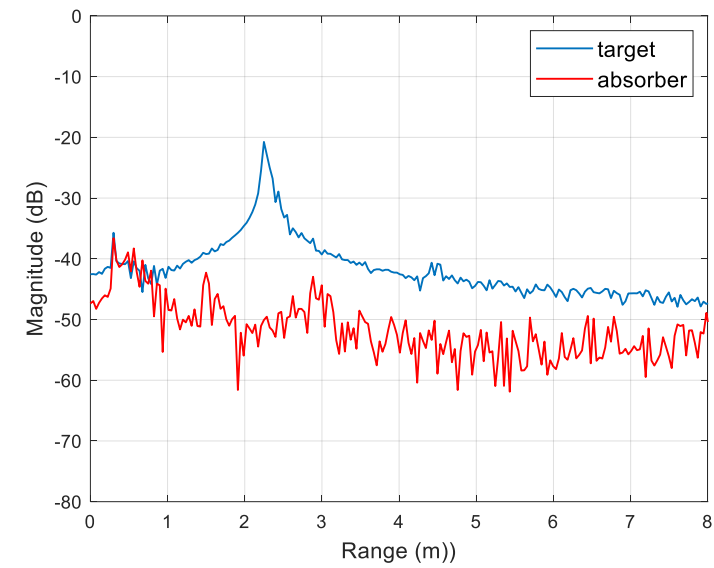

(a)

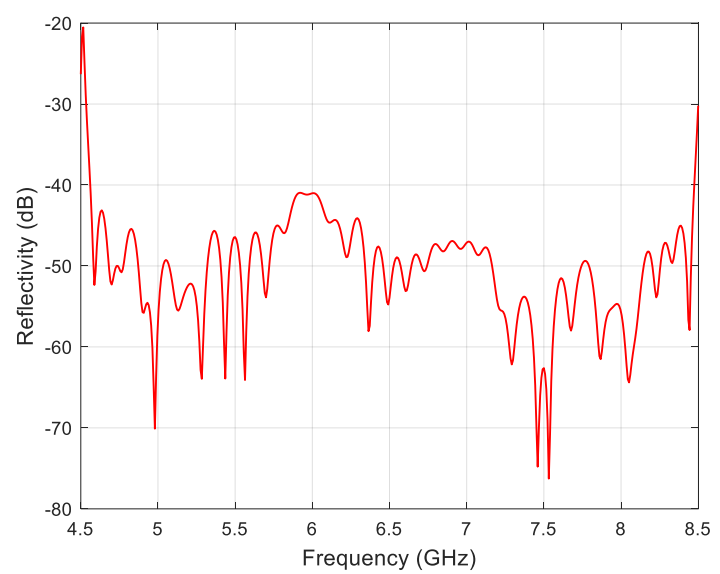

(b)

Figure.3 Results for chamber wall measurements with a plate reference target: (a) range profiles, (b) reflectivity of the absorber.

\subsection{Imaging experiments}

The experiments were intended to determine whether circular ISAR imaging at low frequencies (i.e. up to 22 $\mathrm{GHz}$ ) has a possible application in monitoring concealed objects. For this purpose, various metallic objects either naked or hidden under clothing were selected as targets. The measurements were conducted within the anechoic chamber room $(4 m \times 6.6 m)$ at the Mersin University's MEATRC laboratory.

A total of five consecutive experiments were performed in the quasi-monostatic arrangement of rectangular double-ridged horn antennas (Geozondas GZ0126DRH, $1-26 \mathrm{GHz}$ ) that were spatially separated by a distance of $300 \mathrm{~mm}$ apart. In all experiments, except the fourth one, the frequency of VNA was altered from $4.5 \mathrm{GHz}$ to $8.5 \mathrm{GHz}$ sampling a total of 501 points. In the fourth experiment, however, two dataset were acquired at 801 frequency points within the two frequency ranges; i.e. $1 \mathrm{GHz}$ to $11 \mathrm{GHz}$ and $11 \mathrm{GHz}$ to 22 GHz. Two VNAs were used to cover these frequencies; one is Agilent E5071B (300 kHz to 8.5 $\mathrm{GHz}$ ) and the other is handheld Keysight (Agilent) Fieldfox N9918A ((30 kHz to $26.5 \mathrm{GHz}))$. In all cases, backscatter data were collected by rotating the target under test from 0 to $360^{\circ}$ with $1^{\circ}$ increments and with an antenna to target distance of $R_{0}=2.18 \mathrm{~m}$. It is worth remarking that sampling requirements given in Eqn. (4) and Eqn. (5) were met with the chosen angular and frequency intervals, assuming a maximum target size of around $40 \mathrm{~cm}$.

The data processing steps that we applied prior to imaging are as follows: First, a temporal smoothing function, i.e., Hanning window was applied to the frequency-domain data for sidelobe control. A 1D IFT was then performed to convert the frequency-domain data into time-domain. Next, a time gating was applied to filter out the unwanted echoes falling outside the target region. The resulted signals were then converted again into frequency-domain and calibrated by using Eqn. (6) with the measurement data of background and a conducting sphere with a diameter of $5 \mathrm{~cm}$. Finally, the reflectivity images were reconstructed by a near-field backprojection imaging algorithm. The obtained images and the relevant results are presented in the following.

\subsubsection{Experiment 1: Two cylinders}

For the first experiment, we have selected two small metal (made of cupper) cylinders that have diameters of $2 \mathrm{~cm}$ and lengths of $15 \mathrm{~cm}$ as seen in Fig. 4. After processing the raw data through the processes explained in the previous section, the 2D reflectivity image has been reconstructed by the help of backprojection imaging algorithm, as depicted in Fig. 5(a). The displayed dynamic range is $40 \mathrm{~dB}$. The image shows that each cylinder is well-focused at its true location around wherein the majority of the backscattered energy is confined. Thus, the image features shows an approximate point type scattering, as expected owing to the fact that the cylinders have diameters in the order of wavelength. However, the responses also include ring-shaped defocusing signatures centered at the cylinders' exact locations. These can be well attributed to the layover phenomenon as encountered in 2D imaging of a 3D target under wide azimuthal viewing (Demirci, et al 2015). Moreover, the scattering interactions can also be noticed from the low-level returns located between the two cylinders.

Fig. 5(b) shows the frequency average RCS values of as a function of azimuth angle. The plot has been obtained by first calculating the magnitude squared values of the calibrated frequency-domain data measured at each angle and then averaging the result over the examined frequency band. The presented plot in Fig. 5(b) corresponds to the frequency band of $4.5 \mathrm{GHz}$ to 8.5 GHz. The measured RCS values demonstrate a fluctuation within $-4 \mathrm{~dB}$ and $-12 \mathrm{~dB}$, indicating a high reflection energy over the entire angular views. This is due to isotropic scattering mechanism of cylinder-like targets.

To measure the spatial resolutions, contour plots through the peak of the upper cylinder has been retrieved from the image and sketched as shown in Fig. 5(c). The outer contour corresponds to the $-4 \mathrm{~dB}$, and thus its extent in range and cross-range dimensions gives the -4 $\mathrm{dB}$ resolutions in the respective direction. The distances were found to be same and equal to about $1 \mathrm{~cm}$. This good resolution was expected because of the highresolution capability of full-aperture angular scanning. 
Vol; 5, Issue; 3, pp. 150-159, October, 2020,

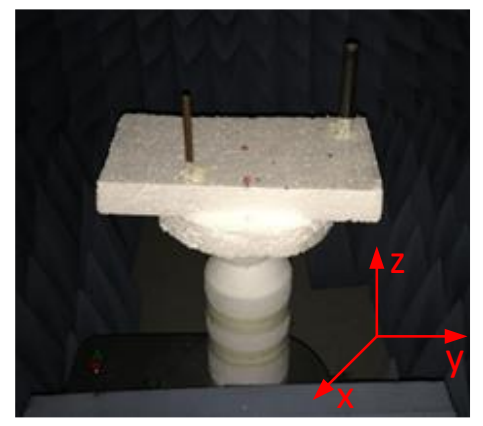

Figure 4. Photograph of the target used in Experiment 1.

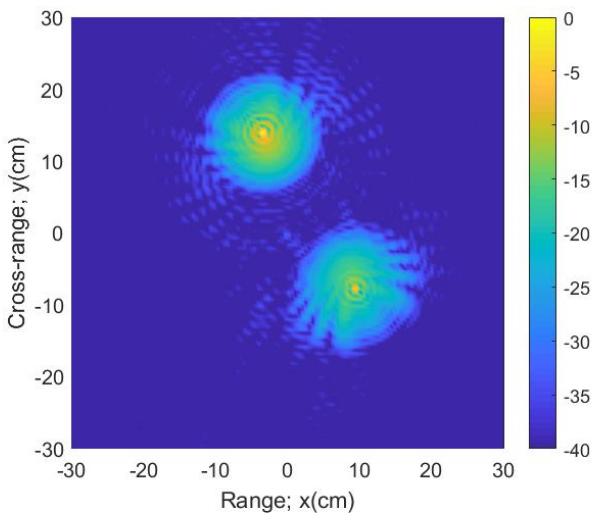

(a)

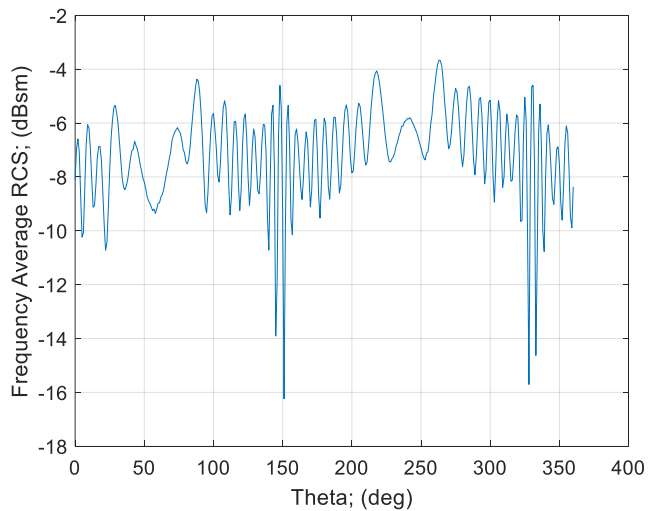

(b)

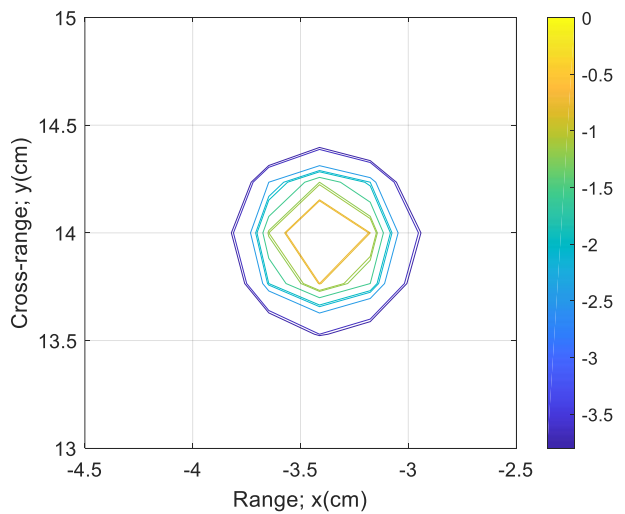

(c)

Figure 5. Results for Experiment 1: (a) Circular ISAR image, (b) frequency average RCS, (c) contour plots around the peak value.

\subsubsection{Experiment 2: Four plates}

Fig. 6 shows the photograph of the target used in the second experiment. The target consisted of four metal plates, placed vertically with respect to the line of sight (LOS) of the radar. The plates on the right and left have larger lengths and widths than the other ones. Fig. 7(a) shows the reconstructed image displayed within the dynamic range of $30 \mathrm{~dB}$. All four plates can be readily identified through their high intensity image signatures. However, the signatures are seen, again, to have layover artifacts shown up vertical lines in this case. The relative sizes of the plates can also be deduced by considering the spatial extent of these signatures. More clearly, the plates shown on the top and bottom of the figure are larger in size, thus they produce wider strong responses.

RCS pattern as the objects is rotated about its vertical axis is shown in Fig. 7(b). It is well known that, RCS of a plate makes a peak when the radar's LOS vector is perpendicular to the plate's surface. When the incident angle deviates from this direction, RCS value decreases, because of the scattering of the wave in different directions other than the direction of the receiving antenna. The plot manifests this fact by exhibiting high RCS values at $0^{\circ}$ and $180^{\circ}$ views.

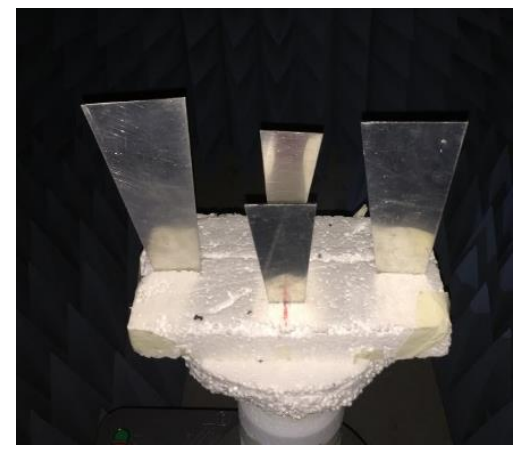

Figure 6. Photograph of the target used in Experiment 2.

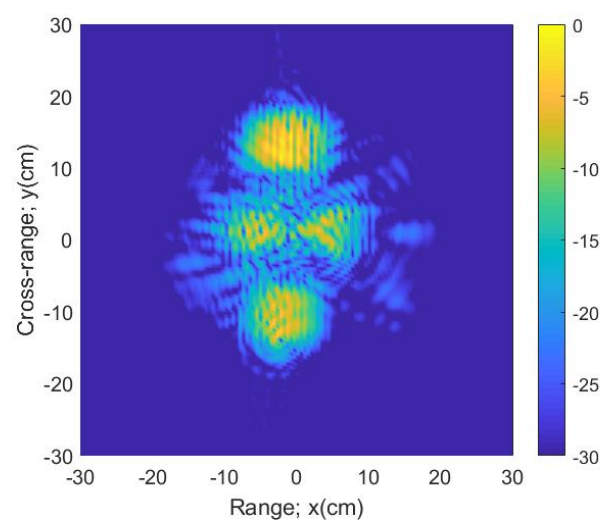

(a) 


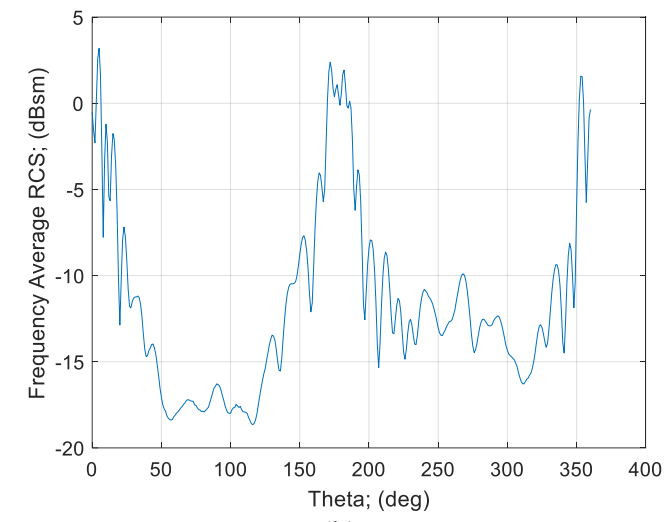

(b)

Figure 7. Results for Experiment 2: (a) Circular ISAR image, (b) frequency average RCS.

\subsubsection{Experiment 3: A screw wrench}

As a third experiment, a more complex object that is a screw wrench with a length of $36 \mathrm{~cm}$ was chosen. Photographs of the target can be seen from Fig. 8, of which the one on the left shows the target's alignment during the experiment. The generated image for this aspect is given in Fig. 9(a). The quality of the image is quite satisfactory, revealing the true size of the target in cross-range direction. However, the size in range direction is not correctly mapped since the energy in this direction is seen to be spread out over a larger region. This may be resulted from the lateral alignment of the target that gives rise to low RCS values. Despite this, some strong scattering centers are shown to be focused at the front and rear parts of the object.

RCS pattern shown in Fig. 9(b) has similar characteristic with those observed for the metal plates, which are both having maximum values at $0^{\circ}$ and $180^{\circ}$. For the current case, the two sides of the wrench faces directly to the radar at these views, thereby giving rise to high backscattered power, as one would expect. The complex structure of the front part, however, is also seen to produce relatively high RCS value at $90^{\circ}$.

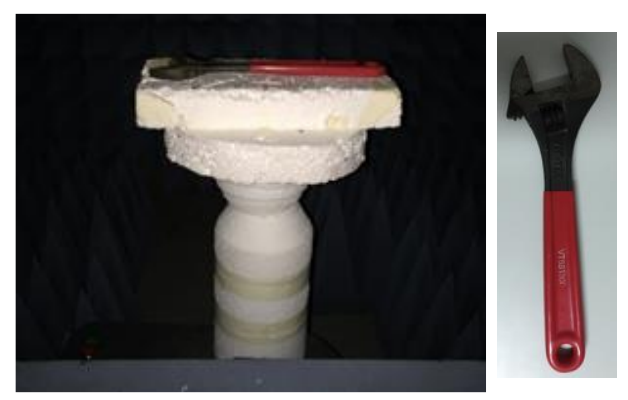

Figure 8. Photograph of the target used in Experiment 3.

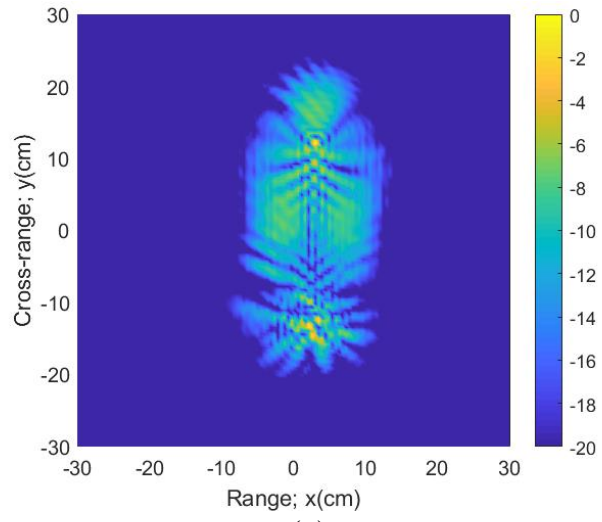

(a)

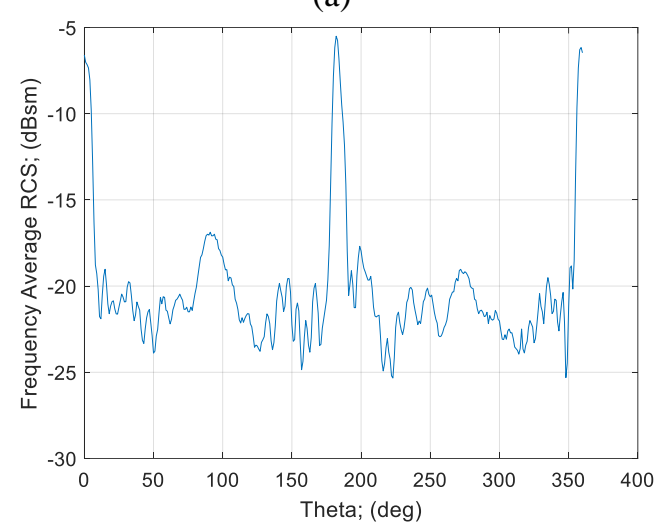

(b)

Figure 9. Results for Experiment 3: (a) Circular ISAR image, (b) frequency average RCS.

\subsubsection{Experiment 4: A handgun}

As for the fourth experiment, backscattering data from a real handgun were gathered within the two different frequency ranges, namely, $1 \mathrm{GHz}$ to $11 \mathrm{GHz}$ and $11 \mathrm{GHz}$ to $22 \mathrm{GHz}$, with intended purpose of investigating the effect of frequency dependence on image signatures. The resulted images are provided in Fig. 9. At first glance, it is noticed that each image successfully displays the target's outline within the $20 \mathrm{~dB}$ dynamic range. Also, the strong returns are seen to come from the gun's magazine, hammer (rear), trigger/trigger guard and muzzle (front) parts in both case. However, the higher-frequency result has shown an overall improvement in image resolution than that of lowerfrequency. This improvement is in good agreement with the Eqn. (5) which implies that the use of higher frequencies improves cross-range resolution for a fixed frequency bandwidth. The gun's several parts are more clearly delineated in the image for $11-22 \mathrm{GHz}$ frequency bandwidth, indicating better visualization and identification capability. This advance may be of particular significance in detecting small, concealed objects. 


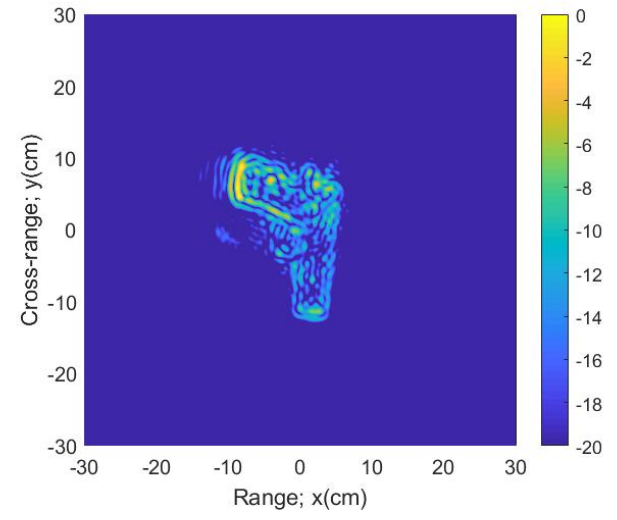

(a)

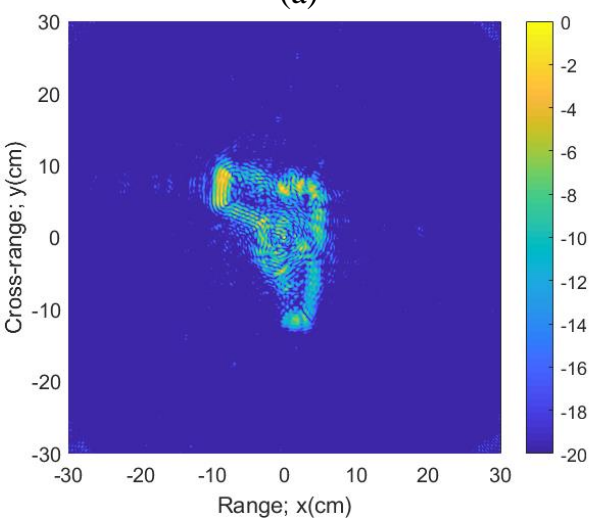

(b)

Figure 10. Circular ISAR images for Experiment 4: (a) $1-11 \mathrm{GHz}$, (b) $11-22 \mathrm{GHz}$ frequency range.

\subsubsection{Experiment 5: A handgun with clothing covering}

It is well known that millimeter waves (e.g., $30 \mathrm{GHz}$ to $300 \mathrm{GHz}$ ) are capable of penetrating clothing thereby detecting small objects under clothing thanks to their small wavelengths (Demirci, et al, 2012). In this experiment, we aimed to provide an initial result for determining to what extent the $\mathrm{C}$-band frequencies could reveal the image of a relatively large target hidden under clothing. For this purpose, the same handgun used in the previous experiment, was scanned in its two different conditions; i.e., one with no cover and the other was covered with a jacket. Note that the bandwidth employed in the experiment was $4 \mathrm{GHz}$ ranging from $4.5 \mathrm{GHz}$ to $8.5 \mathrm{GHz}$ and the standoff distance was $2.18 \mathrm{~m}$, as stated before.

The reconstructed full-aperture ISAR images are shown in Fig. 11. The outline of the handgun can be discerned from both images, though it is less clearly displayed for the covering case. In each situation, the strong scatterers are shown to be located, again, at the gun's magazine, hammer and muzzle parts. As seen from Fig. 11(b) clothing covering presents a distribution of some cluttering (unwanted) echoes positioned around the target region, thus masking target signatures. Nevertheless, it can be stated that that such artifacts, as having relatively low RCS values, do not strongly influence detection performance, since the current target has strong ISAR scattering features that reflect the shape of the target. However, it may not be always possible to overcome this difficulty, if a small-sized or low RCS target is employed.

The reconstructed full-aperture ISAR images are shown in Fig. 11. The outline of the handgun can be discerned from both images, though it is more clearly displayed for the no-covering case. As seen from Fig. 11(b) clothing covering presents a distribution of some cluttering echoes located around the target region, thus masking target signatures. Nevertheless, it is observed that such artifacts do not strongly influence the detection capability, since the current image has strong scattering centers that reflect the shape of the target. However, it may not be always possible to overcome this difficulty, if a small-sized or low RCS target is employed.

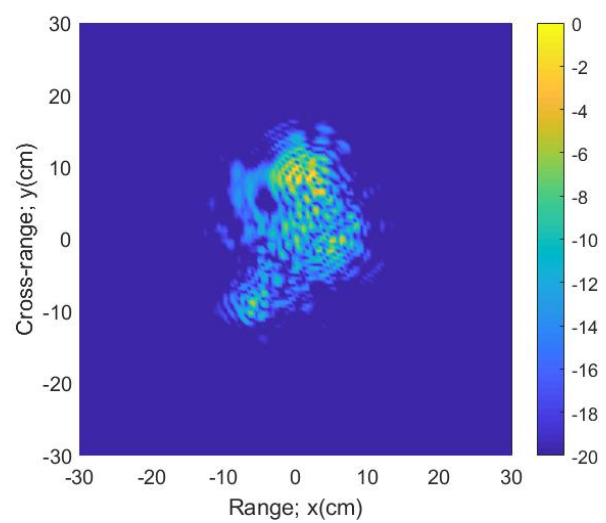

(a)

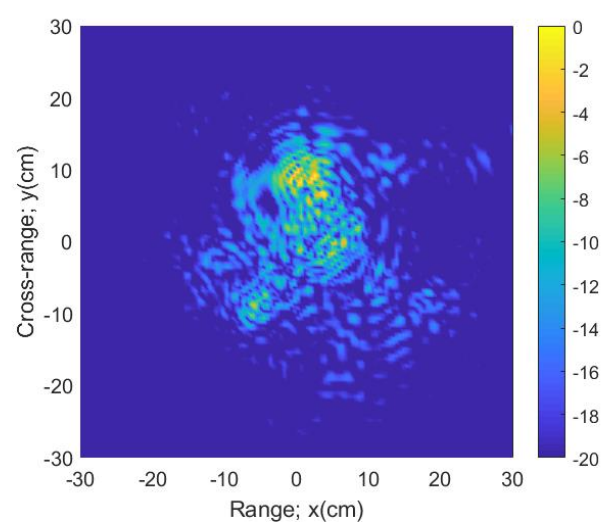

(b)

Figure 11. Circular ISAR images for Experiment 5: (a) gun with no covering, (b) gun covered with a jacket.

\section{CONCLUSION}

In this work, we have presented near-field circular ISAR experiment results that have been conducted in the anechoic chamber at Mersin University's MEATRC facility. Various targets including simple canonic-shaped objects (plates and cylinders) to complex-shaped objects (wrench and hand-gun) have been studied. To compress the ISAR image in angular direction, back-projection type focusing algorithm has been employed. The results from canonical objects have been used to assess the success performance of the imaging study by evaluating the contrast and the resolution metrics of the reconstructed images. Then, realistic targets of a wrench and a handgun were chosen to be objects to be imaged. Reconstructed images have shown the success of the proposed imaging method for imaging small targets (on the order of 
centimeters) in near-field ISAR imaging challenge. As the last study, the concealed object detection application has been examined. It has also successfully demonstrated that the ISAR imaging of a handgun can be easily detected and identified even if it is covered with a jacket.

\section{ACKNOWLEDGEMENTS (OPTIONAL)}

Authors are grateful to the Mersin University's MEATRC management for their support while conducting the experiments.

\section{REFERENCES}

Avşar Aydın, E , Gençoğlan, D. (2016). A bow-tie antenna design for breast cancer detection. The International Journal of Energy and Engineering Sciences, 1 (2), pp. 15-24. Retrieved from https://dergipark.org.tr/tr/pub/ijees/issue/48356/612250

Baird, C., Kersey, W, Giles, R. and Nixon, W. (2005). Exploitation of ISAR imagery in Euler parameter space, Proceedings of SPIE Radar Sensor Technology IX, Vol. 5788, pp. 116-127.

Bertl, S., Dallinger, A. and Detlefsen, J. (2007). Broadband circular interferometric millimetre-wave ISAR for threat detection. Advances in Radio Science, 5, pp. 147-151.

Bertl, S., Dallinger, A. and Detlefsen, J. (2008). Bistatic extension for coherent MMW-ISAR-imaging of objects and humans. Advances in Radio Science, 6, pp. 63-66.

Bertl, S., Dallinger, A. and Detlefsen, J. (2010). Interferometric focusing for the imaging of humans. IET Radar Sonar \& Navigation, 4 (3), pp. 457-463.

Bicer, M. B., Akdagli, A. and Ozdemir, C., (2018). A matching-pursuit based approach for detecting and imaging breast cancer tumor. Progress in Electromagnetics Research M, 64, pp. 65-76.

Çelik A. R., Kurt M. B. and Helhel S. (2019). An experimental performance investigation of an ultrawideband directional antenna in the microwave imaging of breast cancer tumor. Applied Computational Electromagnetics Society Journal, 34, pp.1549-1556.

Cetinkaya, H., Kizilhan. A., Vertiy, A., Demirci, S., Ozdemir, C., Yigit, E., (2011). The millimeter-wave imaging of concealed objects, Spokane-USA, The 2011 IEEE International Symposium on Antennas and Propagation and USNC/URSI National Radio Science Meeting, pp. 228-231.

Chen, C. C. and Andrews, H. C. (1980). Multifrequency imaging of radar turntable data. IEEE Transactions on Aerospace and Electronic Systems, AES-16 (1), pp. 1522.

Cottard, G. and Arien, Y. (2006). Anechoic chamber measurement improvement. Microwave Journal, 49(3), pp. 94.
Currie, N. C. (1989). Radar reflectivity measurement: Techniques and applications. ISBN: 0890063249, Artech House, Norwood, United States.

Demirci, S., Cetinkaya, H., Tekbas, M., Yigit, E., Ozdemir, C. and Vertiy, A. (2011). Back-projection algorithm for ISAR imaging of near-field concealed objects, Istanbul, Turkey, XXXth URSI 2011 URSI General Assembly and Scientific Symposium.

Demirci, S., Cetinkaya, H., Yigit, E., Ozdemir, C. and Vertiy, A. (2012). A study on millimeter-wave imaging of concealed objects: Application using back-projection algorithm. Progress in Electromagnetics Research (PIER), 128, pp. 457-477.

Demirci, S. and Ozdemir, C. (2013). Compressed sensing based imaging of millimeter-wave ISAR data. Microwave and Optical Technology Letters, 55 (12), pp. 2967-2972.

Demirci, S., Yigit, E. and Ozdemir C. (2015). Wide-field circular SAR imaging: An empirical assessment of layover effects. Microwave Opt. Tech. Letters, 57, (2), pp. 489-497.

Detlefsen, J., Dallinger, A., Huber, S. and Schelkshorn, S. (2005). Effective reconstruction approaches to millimeter-wave imaging of humans, New Delhi, India, Proceedings of the 28th General Assembly of International Union of Radio Science, pp. 23-29.

Hong, C. R. and Büyüköztürk, O. (2000). Wideband microwave imaging of concrete for nondestructive testing. Journal of Structural Engineering, 126 (12), pp. 14511457.

Kempf, T., Peichl, M., Dill, S. and Suess, H. (2007). 3D Tower-Turntable ISAR Imaging, Munich, Germany, 2007 European Radar Conference (EuRAD 2007), pp. 114-117.

Knaell, K. and Cardillo, G. P. (1995). Radar tomography for the generation of three-dimensional images. IEE Proceedings - Radar, Sonar and Navigation, 142 (2), pp. 55-60.

Knott, E. F. (2006). Radar cross section measurements. ISBN: 1-891121-55-3, Raleigh, NC, SciTech Publishing.

Mensa, D. L. (1991). High resolution radar cross-section imaging. ISBN: 0890063893, Artech House, Norwood, United States.

Munson, D. C., O’Brien, J. D. and Jenkins, W. K. (1983). A tomographic formulation of spotlight-mode synthetic aperture radar. Proceedings of the IEEE, 71 (8), pp. 917925.

Naseri, M. (2015). Microwave tomography for breast cancer detection, Thesis (M.Sc.). Istanbul Technical University, Institute of Science and Technology.

Ozdemir, C., Kırık, O., Yilmaz, B., (2009). Sub-aperture method for the wide-bandwidth wide-angle inverse synthetic aperture radar imaging, Bursa, Turkey, Int. 
Conference on Electrical and Electronics Engineering ELECO'2009, Bursa, 2, pp. 288-292.

Ozdemir, C. (2012). Inverse synthetic aperture radar imaging with MATLAB algorithms. ISBN: 9780470284841, Wiley Series in Microwave and Optical Engineering, John Wiley \& Sons, Inc., Hoboken, New Jersey.

Sheen, D. M., McMakin, D. L. and Hall, T. E. (2010). Near-field three-dimensional radar imaging techniques and applications. Applied Optics, 49 (19), pp. E83-E93.

Soumekh, D. R. (1999). Synthetic aperture radar signal processing with MATLAB Algorithms. ISBN:
0471297062, John Wiley \& Sons Inc, New York, United States.

To, L., Bati, A., and Hilliard, D. (2009). Radar cross section measurements of small unmanned air vehicle systems in non-cooperative field environments, Munich, Germany, 2009 3rd European Conference on Antennas and Propagation (EuCAP), pp. 3637-3641.

Wehner, D. R. (1994). High resolution radar. ISBN: 0890067279, Artech House, Norwood, United States. 